\title{
Neglected and (re-)emergent infections of the CNS i n low-/middle-income countries
}

\author{
Graciela Cárdenas ${ }^{1}$, Perla Salgado², Enrique Laura-Foronda ${ }^{1}$, Ignacio Popoca-Rodriguez , \\ Rosa Delia Delgado-Hernández ${ }^{2}$, Rafael Rojas ${ }^{3}$, Enrique Palacios ${ }^{4}$ \\ ${ }^{1}$ Neuro-infectious Diseases Department, Instituto Nacional de Neurología y Neurocirugía, \\ Ciudad de México, Mexico; \\ ${ }^{2}$ Neuroimaging Department, Instituto Nacional de Neurología y Neurocirugía, Ciudad de México, Mexico; \\ ${ }^{3}$ Neuroimaging Department, The Beth Israel Deaconess Medical Center, Harvard Medical School, \\ Boston, Massachusett, USA; \\ ${ }^{4}$ Neuroimaging Department, Tulane University, New Orleans, USA
}

Article received 20 August, 2021; accepted 9 November, 2021

\section{SUMMARY}

Low- and middle-income countries (LMIC) have suffered from long-term health system deficiencies, worsened by poor living conditions, lack of sanitation, a restricted access to health facilities and running water, overcrowding, and overpopulation. These factors favor human displacement and deepen marginalization; consequently, their population endures a high burden of infectious diseases.

In this context, the current epidemiological landscape and its impact on health and economic development are not promissory, despite the commitment by the international community to eradicate neglected tropical infections - especially tuberculosis and malaria, by 2030 .

Neglected and (re)-emerging infectious diseases affecting the central nervous system (CNS) are a major public health concern in these countries, as they cause a great morbidity and mortality; furthermore, survivors often suffer from severe neurological disabilities. Herein, we present a retrospective review focused on some neglected and (re)-emerging infectious diseases, including neurocysticercosis, malaria, rabies, West Nile virus encephalitis, tuberculosis, neuroborreliosis, and SARS-CoV-2 in LMIC.
A retrospective review of studies on selected neglected and (re)-emerging infectious diseases in LMIC was performed, including reports by the World Health Organization (WHO) published within the last five years. Data on infection by SARS-CoV-2 were provided by the John Hopkins University Coronavirus Resource Center. CNS neglected and (re)-emerging infectious diseases remain as important causes of disease in LMIC. An alarming increase in the prevalence of malaria, tuberculosis, and cysticercosis is observed in the region, compounded by the recent COVID-19 pandemic. The $\mathrm{WHO}$ is currently supporting programs/efforts to cope with these diseases.

Herein, we highlight the epidemiological burden of some CNS infections in LMIC, and their clinical and neuroimaging features, to facilitate an accurate diagnosis, considering that most of these diseases will not be eradicated in the short term; instead, their incidence will likely increase along with poverty, inequality, and related socioeconomic problems.

Keywords: Neglected, emergent, infections, central nervous system, low/middle income countries.
Corresponding author

Graciela Cárdenas

E-mail: gracielacardenas@yahoo.com.mx;

gcardenas@innn.edu.mx

\section{INTRODUCTION}

Teglected and (re-)emergent infectious diseas1 es are major sources of morbidity and mortality in low-/middle-income countries (LMIC), accounting for one-fifth of all deaths worldwide $[1,2]$. Several factors contribute to increase the burden of infectious diseases, including demo- 
graphic growth, climatic change, human migration, and poverty, along with those inherent to microorganisms (like genetic mutations and antibiotic resistance), which result in an imbalance between pathogens, human hosts, and vectors.

According to United Nations (UN), more than 700 million people or $10 \%$ of the current global population is living in abject poverty, earning less than $\$ 1.90$ a day. This condition is likely to worsen due to the sanitary emergency of COVID-19 and the occurrence of natural disasters (floods, earthquakes, droughts, etc.). In addition, 4 out of 5 people below the international poverty line live in rural areas. Women make up most of this population in poverty in LMIC [3]. There is scarce epidemiological data on (re-)emergent and neglected diseases in LMIC, particularly on diseases affecting the CNS, which pose a persistent health challenge despite the political commitment to fight poverty-related diseases announced by several countries.

Population in LMIC was estimated in about 7.9 billion by 2020, with more than half of them living in poverty or extreme poverty and suffering from a high burden of infectious diseases due to a limited access to health services, basic sanitation, drinking water, education, and adequate housing [4].

\section{METHODS}

A retrospective review of neglected and (re)-emergent infections affecting the CNS, focusing on

Table 1 - Epidemiological data of CNS re-emerging and neglected infectious diseases in the poorest countries in the world.

\begin{tabular}{|c|c|c|c|c|}
\hline Continent & $\begin{array}{c}\text { Malaria } \\
(2015-2019)^{*}\end{array}$ & $\begin{array}{c}\text { Rabies } \\
(2017-2020)^{*}\end{array}$ & $\begin{array}{c}\text { Tuberculosis } \\
(2019)^{*}\end{array}$ & $\begin{array}{c}\text { COVID-19 } \\
(2020-2021)^{* * * *}\end{array}$ \\
\hline $\begin{array}{l}\text { Africa } \\
\text { South Sudan } \\
\text { Burundi } \\
\text { Eritrea } \\
\text { Malawi } \\
\text { Niger }\end{array}$ & $\begin{array}{l}\text { ND } \\
\text { ND } \\
\text { ND } \\
\text { ND } \\
\text { ND }\end{array}$ & $\begin{array}{l}\text { ND } \\
\text { ND } \\
\text { ND } \\
\text { ND } \\
\text { ND }\end{array}$ & $\begin{array}{c}26,000 \\
12,000 \\
2,900 \\
27,000 \\
20,000\end{array}$ & $\begin{array}{c}12,410 \\
20,078 \\
6,839 \\
61.800 \\
6,366\end{array}$ \\
\hline $\begin{array}{l}\text { Asia } \\
\text { Afghanistan } \\
\text { Tajikistan } \\
\text { Nepal } \\
\text { Kirgizstan } \\
\text { Myanmar (Burma) }\end{array}$ & $\begin{array}{l}\text { ND } \\
\text { ND } \\
\text { ND } \\
\text { ND } \\
\text { ND }\end{array}$ & $\begin{array}{l}\text { ND } \\
\text { ND } \\
\text { ND } \\
\text { ND } \\
\text { ND }\end{array}$ & $\begin{array}{c}75,000 \\
8,000 \\
69,000 \\
6,900 \\
167,000 \\
\end{array}$ & $\begin{array}{c}156,284 \\
17,486 \\
813,327 \\
181.327 \\
500,925 \\
\end{array}$ \\
\hline $\begin{array}{l}\text { America } \\
\text { Haiti } \\
\text { Nicaragua } \\
\text { Venezuela } \\
\text { Honduras } \\
\text { Bolivia } \\
\end{array}$ & $\begin{array}{c}77,663 \\
48,671 \\
1,980,771 \\
10,003 \\
31,758 \\
\end{array}$ & $\begin{array}{c}8 \\
\text { ND } \\
\text { ND } \\
\text { ND } \\
4 \\
\end{array}$ & $\begin{array}{c}19,000 \\
2800 \\
13,000 \\
3000 \\
12,000 \\
\end{array}$ & $\begin{array}{c}23,960 \\
16,422 \\
407,151 \\
385.375 \\
26.978 \\
\end{array}$ \\
\hline $\begin{array}{l}\text { Europe } \\
\text { Moldova } \\
\text { Ukraine } \\
\text { Georgia } \\
\text { Azerbaijan } \\
\text { Armenia }\end{array}$ & $\begin{array}{l}\text { ND } \\
\text { ND } \\
\text { ND } \\
\text { ND } \\
\text { ND }\end{array}$ & $\begin{array}{l}\text { ND } \\
\text { ND } \\
\text { ND } \\
\text { ND } \\
\text { ND }\end{array}$ & $\begin{array}{c}3,000 \\
32,000 \\
2,800 \\
5,900 \\
680\end{array}$ & $\begin{array}{c}339,114 \\
3,073,125 \\
721,388 \\
532,570 \\
309,397\end{array}$ \\
\hline $\begin{array}{l}\text { Oceania } \\
\text { Kiribati } \\
\text { Salomon Island } \\
\text { Papua New Guinea } \\
\text { Micronesia } \\
\text { Vanuatu }\end{array}$ & $\begin{array}{l}\text { ND } \\
\text { ND } \\
\text { ND } \\
\text { ND } \\
\text { ND }\end{array}$ & $\begin{array}{l}\text { ND } \\
\text { ND } \\
\text { ND } \\
\text { ND } \\
\text { ND }\end{array}$ & $\begin{array}{c}510 \\
450 \\
39,000 \\
86 \\
120\end{array}$ & $\begin{array}{c}2 \\
20 \\
29,813 \\
1 \\
\text { ND }\end{array}$ \\
\hline
\end{tabular}

ND. No data available; *World Health Organization Reports; ${ }^{* *}$ John Hopkins University Coronavirus Resource Center. 
LMIC, is herein reported. A search was performed on the Neglected, Tropical, and Vector-Borne Disease Report by the WHO. In addition, epidemiological data on SARS-CoV-2 were retrieved from the John Hopkins Coronavirus Resource Center. In Table 1 the current available epidemiological data of these diseases in the poorest countries of each continent is summarized.

This work is aimed to highlight available epidemiological data, as well as the clinical and neuroimaging features of selected diseases (neurocysticercosis, malaria, rabies, West Nile virus, tuberculosis, borreliosis, and neuro-COVID), to assist clinicians in making a more accurate diagnosis.

\section{Neurocysticercosis}

Neurocysticercosis (NC), caused by the larval stage of Taenia solium, is the most frequent helminthic disease affecting the CNS. The parasite can establish in tissues and organs like the eyes, skin, and muscles, in addition to the CNS. In this latter location, the helminth can be lodged in the brain parenchyma, subarachnoid space, intraventricular space, or in the spinal cord [5].

NC has a significant impact on public health and on the global economy, being the top foodborne parasitic disease according to WHO. NC accounts for one-third of all epilepsy cases in many endemic areas, but this rate can rise to $70 \%$ in highrisk communities. NC mainly affects developing countries in Africa, Asia, and Latin America. In 2015, the WHO reported the loss of 2.8 million disability-adjusted life-years (DALYs) due to NC, and the total number of cases was estimated in 2.56-8.30 million [6].

The clinical manifestations of NC are very heterogeneous and may include epilepsy, intracranial hypertension, and even hydrocephalus, depending on the size and the number of cysticerci, as well as their location in brain tissues (either parenchymal or extraparenchymal) [7].

The radiological presentation of NC is heterogeneous and varies according to the parasite degenerating stage. Neuroimaging tools like MRI and $\mathrm{CT}$ are crucial to define the number, location, size, and degenerating stage of parasites, as well as the host immune response [8].

Neuroimaging findings in NC are exemplified at Figure 1. Serological tests like the enzyme-linked immunosorbent assay (ELISA) are particularly valuable when neuroimaging is not available,

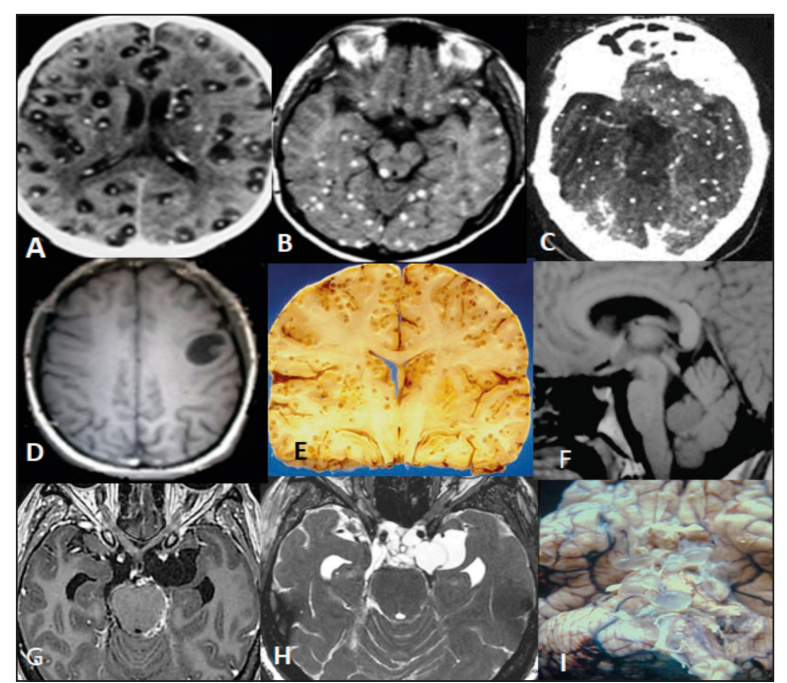

Figure 1 - Neurocysticercosis.

A) Axial CT shows parenchymal neurocysticercosis with multiple vesicular lesions, some of them with visible scolex.

B) Axial MRI, contrast-enhanced T1-weighted sequence shows parenchymal miliary colloidal lesions with gadolinium enhancement.

C) Axial CT shows multiple parenchymal calcified lesions.

D) Axial MRI shows a solitary vesicular lesion with a prominent scolex lodged at left frontal region.

E) Brain coronal pathological specimen shows vesicular miliary neurocysticercosis.

F) Sagittal MRI shows a solitary colloidal cysticercus in the fourth ventricle.

G) Axial MRI T1-weighted shows multiple vesicular lesions at subarachnoid space of the base.

H) Axial MRI on FIESTA sequence shows subarachnoid vesicular lesions.

I) Ventral side of a brain pathological specimen shows multiple vesicular lesions at the basal cisterns.

while the detection of the HP10 antigen in cerebrospinal fluid (CSF) and serum is useful when extraparenchymal areas of the brain are involved (cisternal, intraventricular, and/or subarachnoid space) $[9,10]$.

Although the incidence of NC has decreased in recent years, it is still an important health and economic concern in the LMIC, due to the losses it causes in the pork industry and in the economy of small farmers. Both problems should be carefully considered when planning NC eradication efforts.

\section{Malaria}

The most prevalent parasitic disease in the world, malaria remains a major threat to public health in 
Figure 2 - Malaria.

A) Axial MRI T2-weighted sequence shows multiple hyperintense lesions in the hemispheric white matter.

B) and C) Axial MRI on FLAIR sequences show hyperintense lesions in the in the hemispheric white matter and basal ganglia nuclei.

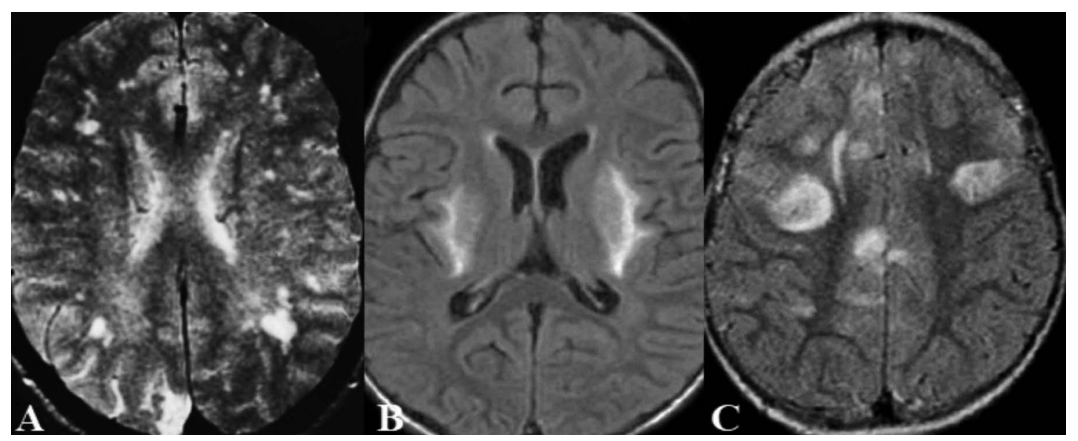

tropical and subtropical regions. Several protozoan species of the genus Plasmodium are known to cause the human disease. The population segments at highest risk of a fatal outcome include children under 5 years and pregnant women infected by Plasmodium falciparum. Plasmodium vivax is the predominant species in sub-Saharan Africa, where infections by Plasmodium ovale and Plasmodium malariae have also been reported, although with a lesser prevalence. On the other hand, Plasmodium knowlesi, Plasmodium cynomolgi, and Plasmodium brasilianum are primarily zoonotic parasites, mostly infecting primates [11, 12].

In 2019, 229 million malaria cases were reported in 87 endemic countries, most of them in Africa, followed by Southeast Asia and the Eastern Mediterranean regions. During 2019, almost 51\% of cases occurred in Nigeria, Democratic Republic of the Congo, Uganda, Mozambique, and Niger, while deaths clustered in Nigeria, Democratic Republic of the Congo, United Republic of Tanzania, Burkina Faso, Mozambique, and Niger [13]. Fortunately, some countries around the world were certified as malaria-free by the WHO in recent years: El Salvador, Argentina, and Paraguay (Americas); Algeria (Africa); Armenia (Europe); United Arab Emirates, Turkmenistan, Kirgizstan, and Sri Lanka (Asia) [13, 14].

Malaria is transmitted by various mosquito species of the genus Anopheles. Several factors are known to be related to the pathogenesis of cerebral malaria, including microvascular obstruction by P. falciparum-parasitized red blood cells, cytokine storm, microvascular thrombosis, and endothelial dysregulation [11, 12]. The most frequent clinical manifestations are retinopathy and brainstem alterations, while general complaints include asthenia, fever, chills, headache, myalgia, abdominal pain, and cough [15]. The diagnosis should be confirmed by a blood smear and antigen detection (HRP-2) or PCR. In severe cases, a neuro-ophthalmologic analysis is mandatory to search for papilledema and retinal hemorrhages [11]. Neuroimaging findings (Figure 2) in cerebral malaria include hyperintense cortical or white matter lesions on T2-weighted images (posterior reversible encephalopathy syndrome), as well as petechial hemorrhages in the gray matter; in these cases, susceptibility-weighted angiography (SWAN) is of great value for diagnosis, as well as gradient echo pulse sequences [16].

To improve advances in the control is essential to improve the current protocols to remove anopheline habitats and hatcheries (filamentous green algae) in affected communities, and to cut peridomiciliary vegetation (the resting place for adult vectors) in high-risk areas.

\section{Rabies encephalitis}

Rabies, a life-threatening disease caused by a neurotropic virus of the family Rhabdoviridae that affects mammals and humans, is still an important public health problem worldwide, with a high mortality [17]. Globally, the annual number of human deaths due to rabies by dog bite was estimated in 59,000 [18]. Dog-mediated rabies was eradicated in Western Europe, some countries of the Americas (Canada, United States, Mexico, and Brazil), as well as in Japan, Australia, and most Pacific Island nations.

In Asia, rabies is still a major disease, with an estimated 35,172 human deaths per year, while it causes an estimate of 21,476 deaths yearly in Africa. About $60 \%$ of rabies-related deaths in Asia and $35 \%$ of global deaths due to rabies occur in India $[18,19]$. 
The pathophysiology of human rabies has five stages: incubation, prodromal, acute neurologic affection, coma, and death. The incubation period depends on the distance between the entrance site of the virus (bite site) and the head. Lesions proximal to the head show a shorter incubation period, but in most cases $(60 \%)$ it takes from one to three months. Rabies virus can reach the CNS through a centripetal or retrograde pathway from the inoculation site [17]. The early clinical signs of rabies include fever, headache, vomiting, and irritability. Other less frequent complaints are paresthesia and neuropathic pain. In the neurologic stage, the patients develop encephalomyeloradiculitis and autonomic dysfunction signs like hypersalivation, excessive sweating, piloerection, pupillary alteration, and even hemodynamic instability, arrhythmias, priapism, and neurogenic pulmonary edema. Two main forms of rabies have been described: encephalitic (furious) in $67 \%$ of cases, and paralytic in 33\%. The coma stage, lasting from 5 to 14 days, is characterized by autonomic instability and multiorgan dysfunction. The brainstem, diencephalon, and hypothalamus are the most affected regions [20, 21]. The pathognomonic clinical signs of rabies are hydrophobia, aerophobia, and hypersalivation [22]. MRI findings (Figure 3) are characterized by hyperintense lesions in T2weighted images, particularly in the spinal cord, brainstem, basal ganglia, hippocampus, and pa- rietal/frontal lobes. In post-mortem studies, necrosis involving both white and gray matter in the basal ganglia, cortex, and brainstem, along with Negri bodies (eosinophilic, sharply outlined bodies in the cytoplasm of affected neurons) is pathognomonic [22].

Significant progress has been made in canine vaccination coverage and accessibility to post-exposure prophylaxis, particularly in Latin America and the Caribbean, where a significant decrease in the number of human and dog rabies cases has been recorded; in fact, rabies cases were only reported in 8 countries during 2013 and 2016. However, there is an urgent need to implement rabies control programs in other endemic regions to reduce mortality rates. The global community has set the goal of eradicating human deaths from dog-mediated rabies by 2030 [19].

\section{West Nile virus encephalitis}

West Nile encephalitis (WNV) is a globally distributed infectious disease. Its transmission depends on several mosquito species (mostly Culex spp.) and affects birds in its enzootic cycle. WNV cases were reported in many regions around the world during the last 50 years. Major outbreaks have occurred in Greece, Israel, Romania, Russia, the United States, and other countries in the path of major bird migratory routes. Over 48,000 cases, 24,000 neuroinvasive cases, and 2300 deaths have

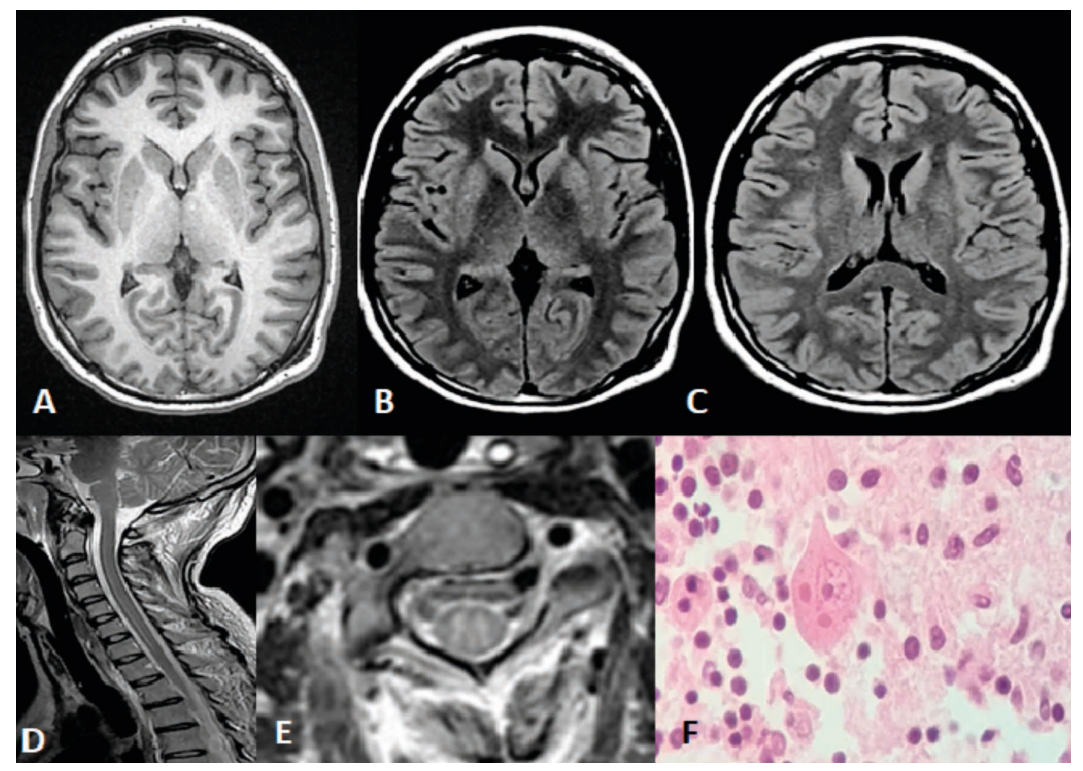

Figure 3 - Rabies.

A 24-year-old woman with a history of being bitten by a grey fox (Urocyon cinereoargenteus) three weeks before hospital admission. Upon emergency admission,

A) MRI T1-weighted sequence shows no apparent lesions.

B) and C). FLAIR sequences show faint lesions at basal ganglia (globus pallidus).

D) Sagittal MRI of the cervical spinal cord shows intramedullary longitudinal hyperintense lesion.

E) Axial MRI at a cervical level shows hyperintense lesion in the gray matter.

F) Post-mortem hematoxylin-eosin-stained section at the cerebellar cortex shows eosinophilic neuronal cytoplasmic inclusions (Negri bodies). 
Figure 4 - West Nile virus encephalitis.

A) Axial MRI T1-weighted shows bilateral hyperintensities at the basal ganglia, more prominently at the left side.

B) Axial contrast-enhanced MRI bilateral enhancement, nodular, at the left side of basal ganglia.

C) Axial MRI on diffusion sequence shows bilateral hyperintensities at the basal ganglia.

D) Sagittal MRI at thoracic level shows an intramedullary longitudinal hyperintense lesion.

E) Axial MRI contrast enhanced T1 sequence at a lumbar level shows enhancement and thickening of the spinal roots the cauda equina.

F) Axial pathological specimen of the spinal cord shows right side hemorrhagic lesion.ì

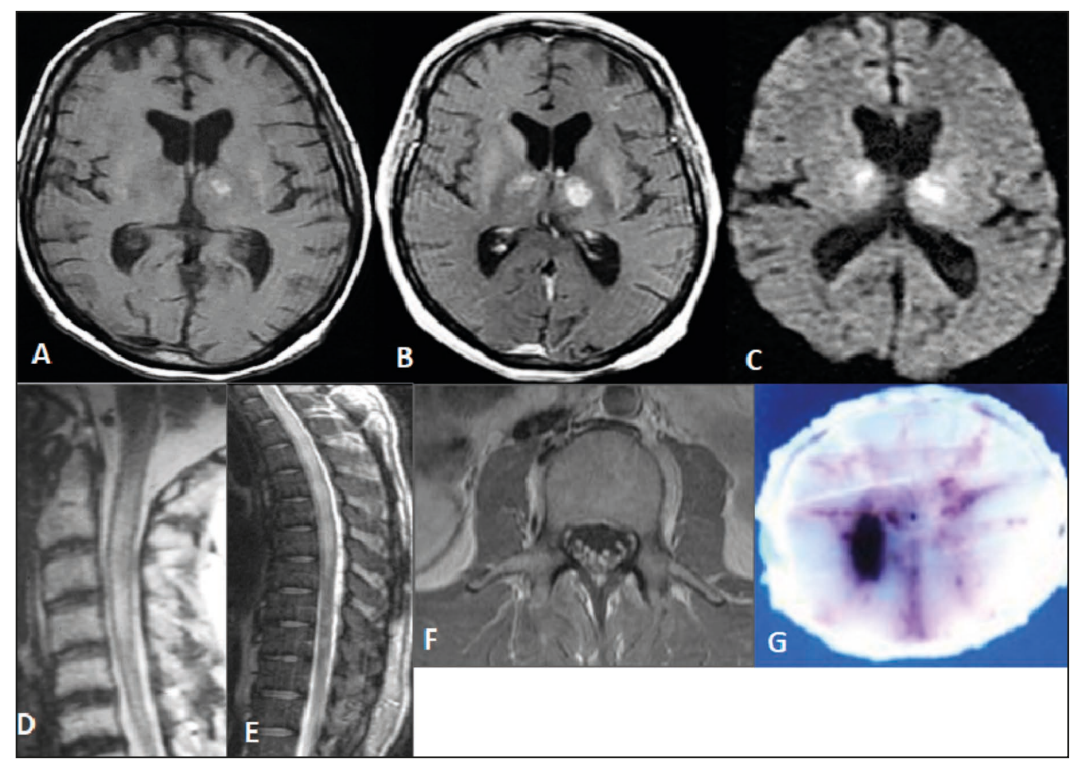

been reported since its emergence in the Americas, in 1999 [23, 24]. Most infected patients (80\%) are asymptomatic, while $15-20 \%$ develop a mild disease (WNV fever), and less than 1\% show neurological signs like encephalitis, meningitis, meningo-encephalitis, ataxia, and polyradicultis [25]. When performed in the viremic stage, molecular assays like real-time quantitative polymerase chain reaction (RT-qPCR) in blood, urine, or CSF seem to be specific, but this diagnostic approach is not frequently used because viremia is very short-lived. In this context, serological assays performed 5 to 8 days after the onset of clinical symptoms by detecting IgM antibodies (ELISA) in serum or CSF samples are useful. The major drawback of serologic diagnosis is the cross-reactivity with other viral species of the genus Flavivirus [26].

Neuroimaging studies (Figure 4), particularly MRI, show alterations in at least two-thirds of WNV-infected patients, including inflammatory hyperintense changes in the white matter or bilateral basal ganglia (thalamus) on T2-weighted and FLAIR sequences. Leptomeningeal enhancement is observed in $30 \%$ of patients, while intramedullary hyperintensities in the spinal cord, involving cauda equina, are also commonly found [26].

WNV infection, particularly the neuroinvasive disease, is an important cause of morbidity and mortality worldwide, and the implementation of adequate nationwide preventive programs is required to control the disease. To help controlling vector-borne diseases like DENV, ZIKV, and Chikungunya virus (CHIKV) in LMIC, it is mandatory to monitor the density of transmitting insects and control their population in all development stages, particularly the aquatic and adult stages.

\section{Central nervous system tuberculosis}

Mycobacterium spp. are acid-alcohol fast bacilli. Mycobacterium tuberculosis and Mycobacterium bovis are the main etiologic agents of tuberculosis (TB) in humans [27]. TB remains an important cause of death worldwide. Globally, an estimated 10 million people were infected with the TB bacillus in 2019, while about 1.2 million TB-related deaths in the HIV-negative population and 215,000 deaths in HIV-infected patients occurred in that year. With respect to geographical regions, most TB cases were reported in South-East Asia $(44 \%)$, Africa $(25 \%)$, and Western Pacific (18\%). A smaller proportion was reported in Eastern Mediterranean $(8.2 \%)$, the Americas $(2.9 \%)$, and Europe $(2.5 \%)$ [28].

Currently, TB burden is concentrated in 30 countries; two-third of all cases occurred in eight countries: India (26\%), Indonesia (8.5\%), China (8.4\%), Philippines (6\%), Pakistan (5.7\%), Nigeria (4.4\%), Bangladesh (3.6\%), and South Africa (3.6\%) [28]. Extrapulmonary TB, including CNS TB, is due 
to hematogenous or lymphatic dissemination of bacteria from the lungs. It often causes tuberculous meningitis, tuberculomas, and abscesses. Hydrocephalus is the main clinical sign, usually associated to vasculitis, cranial neuropathy, and neuropsychiatric symptoms [29].

A definitive TB diagnosis is difficult, since it requires acid-alcohol fast bacilli isolation on CSF cultures, nucleic acid detection by PCR, or histopathological confirmation. In this context, Marais et al. proposed a useful set of diagnostic criteria. Adenosine deaminase (ADA) levels $>7 \mathrm{UI} / \mathrm{mL}$ in CSF are indicative of TB infection [30]. ADA quantification has a higher sensitivity than commercial PCR assays (90.5\% and $86.5 \%$, respectively), and it is less expensive [31].

Neuroimaging findings (Figure 5) in CNS TB include meningeal enhancement, infarcts, hydrocephalus, and vasculitis [32]. A study including imaging results of 559 patients (452 MRI and 209 CT) over a 10-year period was published recently. Initial CT scans revealed incidence rates of $12 \%$ for tuberculoma, 25\% for infarct, $14 \%$ for meningeal enhancement, and $40 \%$ for hydrocephalus, while MRI revealed tuberculoma in $40 \%$ of patients, infarction in $27 \%$, meningeal enhancement in $41 \%$, and hydrocephalus in $26 \%$ [33].

Current WHO efforts to implement public health programs for early TB detection in at-risk populations are crucial to prevent the emergence of resistant strains, to control the disease, and to its eventual eradication by 2030 [28].

\section{Neuroborreliosis}

This tick-borne zoonotic infection constitutes a significant global public health concern. Borrelia burgdorferi sensu lato is the causal agent of Lyme disease [34]. In Europe, approximately 85,000 cases are reported annually [35]. According to the CDC, a total of 275,589 Lyme disease cases were reported (208,834 confirmed and 66,755 probable cases) in the United States in 2008-2015 [36].

In a Mexican cohort of children and adult in Mexico, a total of 606 patients were included. The most frequent neurological manifestations were cranial neuropathy in 222 , facial palsy in 173 , polyradiculoneuropathy in 110, encephalomyelitis in 77, and meningitis in 24 . In addition, inflammatory alter-

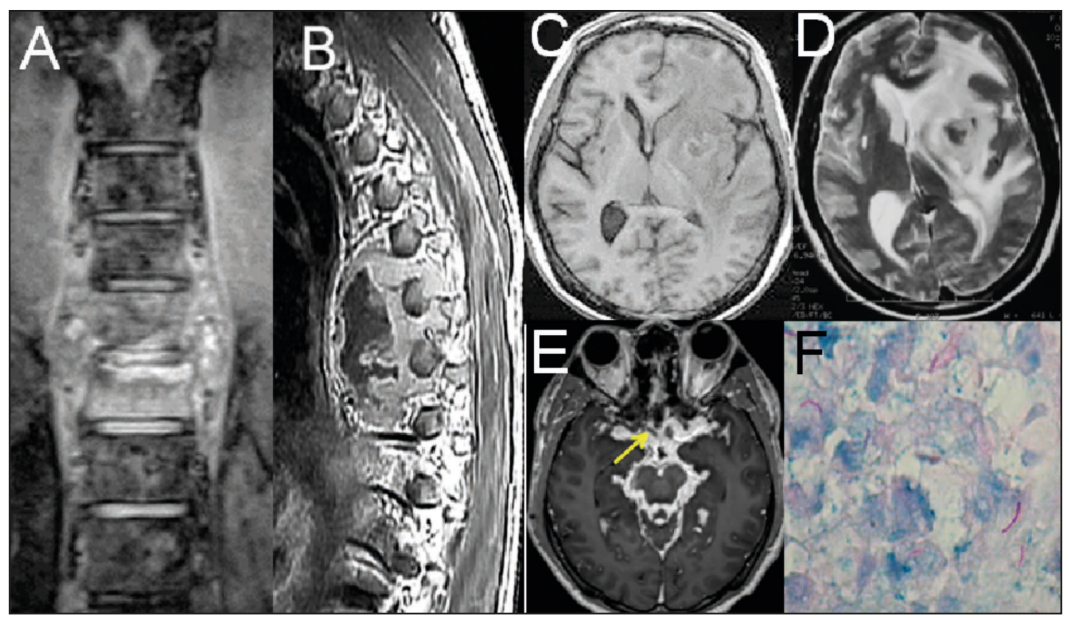

Figure 5 - Tuberculosis.

A) Coronal MRI at a thoracic level shows an abscess with intervertebral disc involvement (spondylodiscitis/Pott disease).

B) Sagittal MRI imaging at a thoracic level shows an abscess involving two vertebral bodies (spondylodiscitis / Pott disease).

C) Axial MRI T1-weighted shows a heterogeneous, hypointense left-side basal ganglia lesion.

D) Axial MRI T2-weighted shows an isointense capsule and important vasogenic edema involving left frontal and insular regions.

E) Axial contrast-enhanced MRI T1-weighted shows basal meningitis (arachnoiditis) and a mycotic aneurism at the right middle cerebral artery.

F) Alcohol-acid resistance bacilli are observed in the purulent aspiration of an abscess. 
ations in the CSF like pleocytosis and high protein content were more common in the adults [37].

Early clinical manifestations include a skin lesion called erythema migrans (EM), which occurs 3-30 days after infection onset. In adults, the predominant lesion sites were the limbs and trunk, while in children they were head and neck. In untreated patients, EM spontaneously resolves within days or weeks. Neurological symptoms (neuroborreliosis) include lymphocytic meningitis, cranial neuropathy, and radiculitis, but neurobehavioral alterations are common [38].

Diagnosis is usually clinical and requires either a combined antecedent of exposure and signs with a positive predictive value, like EM, or laboratory support (a positive two-tiered serologic testing). In addition, marked alterations in CSF cytochemical parameters and intrathecal production of specific antibodies are usually observed in neuroborreliosis [39]. Neuroimaging studies (Figure 6) could be normal even in symptomatic patients, but periventricular or subcortical hyperintensities in T2 and FLAIR sequences have been described, as well as cranial nerve and radicular contrastenhancement [40].

\section{SARS-CoV-2 and neuro-COVID}

SARS-CoV-2, a $\beta$-coronavirus whose genome is formed by a single-stranded RNA chain, has proved to be capable of crossing the interspecies barrier and cause human infection. All coronaviruses can induce both respiratory and intestinal diseases (either inoculated by airborne droplets or through a fecal-oral route) [41-43].

$\beta$-coronaviruses, including SARS-CoV-2, use the spike surface protein (S) to bind the host ACE2 receptor or the cellular serine proteases TMPRSS2 and TMPRSS4 for S protein priming; therefore, they exhibit a high tropism to nervous tissues (neurotropism and neuroinvasiveness) [44-46]. According to the John Hopkins University Coronavirus Resource Center, from the start of the pandemic until November 1, 2021, there were $247,447,923$ cases in the world, and 5,011,786 deaths in the world [47]. Currently, there are $93,711,700$ confirmed cases in the Americas, $77,231,883$ in Europe, 44,005,474 in Southeast Asia, 16,382,071 in the Eastern Mediterranean, 9,456,219 in Western Pacific, and 6,154,163 in Africa [48].

While the clinical presentation of COVID-19 is usually characterized by respiratory alterations ranging from flu-like symptoms to pneumonia, it is now considered as a multisystem disease. Diagnosis requires either a positive RT-PCR SARSCoV-2 test or the combination of an abnormal chest CT and a positive ancillary test (lymphopenia/neutrophilia, elevated liver enzymes, and elevated inflammatory biomarkers, including CRP, ferritin, D-dimer, and CPK levels) [49-51].

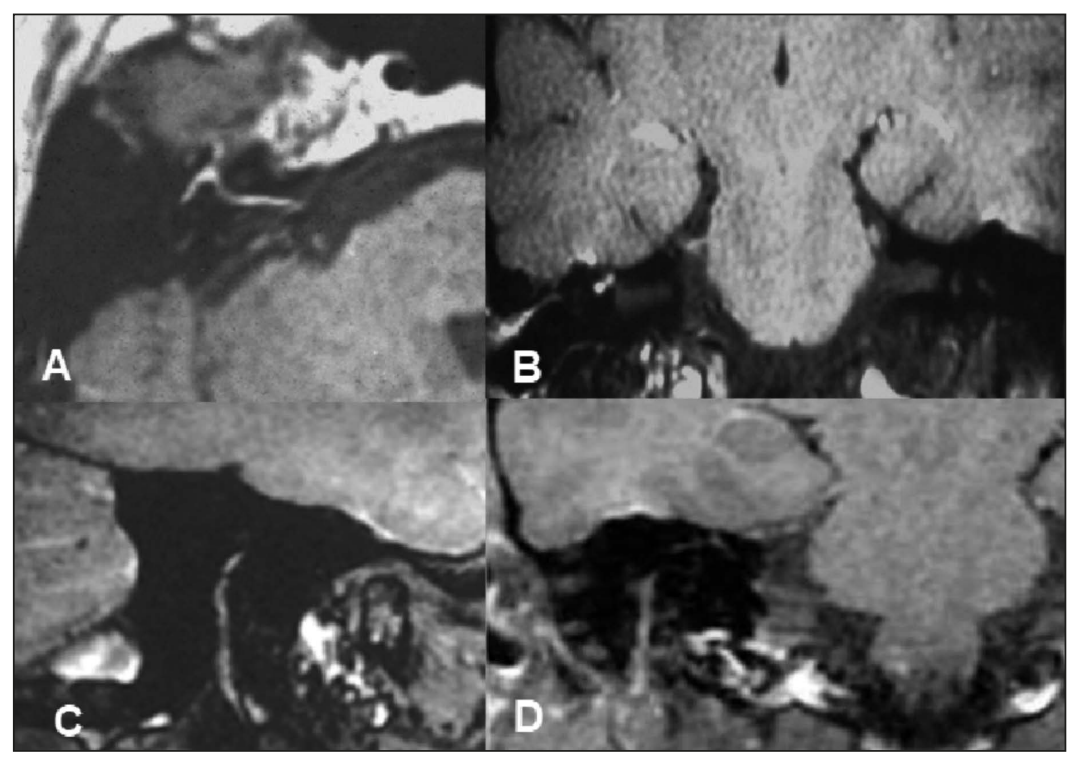

Figure 6 - Lyme disease.

A) Axial contrast-enhanced MRI at the level of intracanalicular, genicular ganglion and tympanic portions of the facial nerve. Clinically, patients presented an atypical Bell's palsy.

B) Coronal contrast-enhanced MRI shows limbs of the facial nerve.

C) and D) Sagittal and coronal contrast-enhanced MRI shows the descending portion of the facial nerve. 
The neurological manifestations of COVID-19 are heterogeneous and can be observed before, during, or after the acute infection. These manifestations can be divided into three categories [52-54]:

1) Central nervous system: headache, dizziness, mental alterations, encephalitis, ataxia, seizures, and cerebrovascular diseases (Figure 7, A-L);

2) Peripheral nervous system: cranial nerve alterations (Figure 7 M-S) (anosmia, hyposmia, dysgeusia, and visual alterations), neuropathic pain, and Guillain-Barré syndrome;

3) musculoskeletal: myopathy, myalgia, and fatigue.
A wide spectrum of neuroimaging findings has been observed in this infection. Lin et al. described a retrospective cohort of adult COVID-19 patients including 2054 cases of whom only 278 underwent brain CT or MRI imaging. From the latter, $58(21 \%)$ showed findings compatible with acute or subacute neurological disorders, being cerebrovascular disease the most frequent one (infarction, $11 \%$; parenchymal hematoma, $3.6 \%$; critical illness-associated microhemorrhage, $1.1 \%$; nontraumatic subdural hemorrhage, $1.1 \%$; and nonaneurysmal subarachnoid hemorrhage, $0.7 \%$ ) followed by cranial nerve abnormalities, $2.2 \%$, and posterior reversible encephalopathy (PRES), 1.1\%

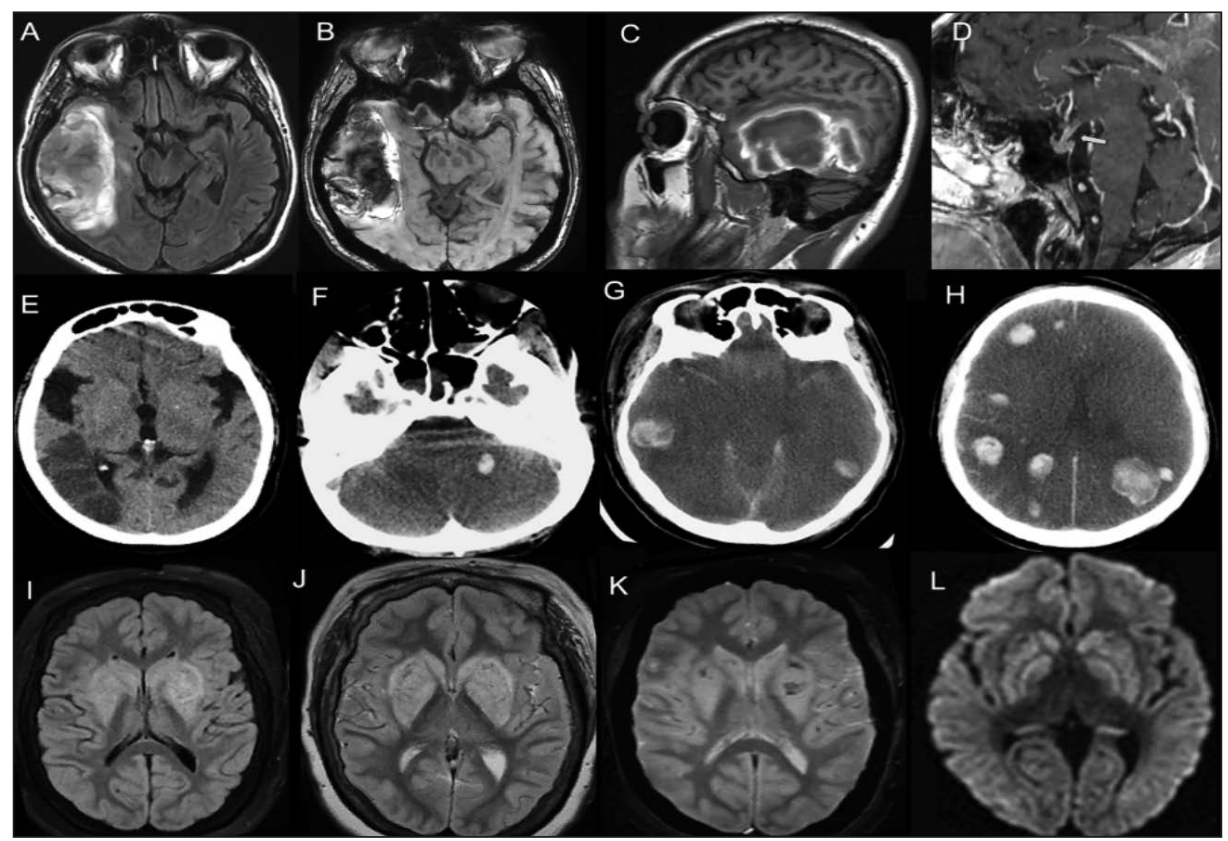

Figure 7 (A-L) - Neuro-COVID-19.

Cerebrovascular manifestations

Case 1. (A to D) A 37-year-old female which developed atypical pneumonia and SARS-CoV-2 infection was confirmed by a positive RT-PCR. After two-weeks of respiratory symptoms, suddenly complained of headache and left hemiparesis. In the MRI, it was observed on FLAIR sequence a hyperintense right temporal lesion (A), hypointense on SWAN sequence (B) which involved superior, media and inferior gyrus, highly suggested of a cerebral infarction with hemorrhage transformation. After the administration of gadolinium, a cortical gyral enhancement (C) and thickening of the infundibulum was observed (D, long yellow arrow).

Case 2. (E) A 58-year-old woman, with developed atypical pneumonia by COVID-19, she suddenly developed right anisocoria. CT scan showed ischemic stroke involving the temporal posterior region by obstruction of the right middle cerebral artery without mass effect.

Case 3. (F to H) A 48-year male with atypical pneumonia by COVID-19, he developed severe hypoxia with neurological worsening characterized by fixed symmetrical pupils. CT scan showed multiple hyperdense lesions (infra and supratentorial hemorrhages), associated with cytotoxic edema as well as hyperdensity in both M1 segments of middle cerebral artery.

Case 4. (I to L) A 70-year-old woman with COVID-10 pneumonia which developed hyperintense (anoxic) brain lesions observed in T1, FLAIR, diffusion, and SWAN. 

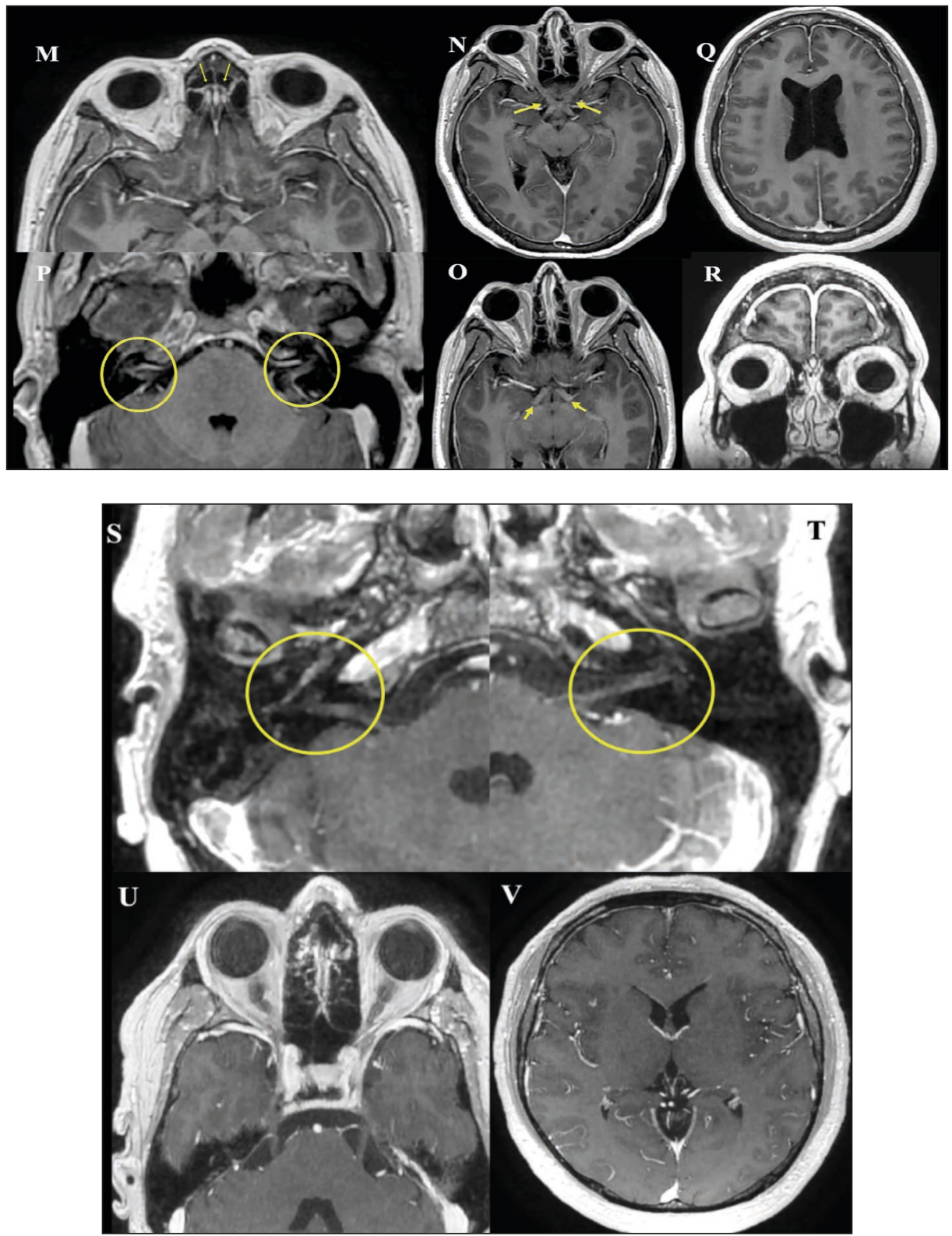

Figure 7 (M-V) - Neuro-COVID-19.

Nerve and pachymeningeal enhancement manifestations

Case 5. (M to R) A 29-year-old female which was hospitalized by a psychotic episode and after 14-days, she developed dry cough and atypical pneumonia by SARS-CoV-2. In the MRI, after gadolinium administration it was observed post-contrast enhancement in the olfactory grooves (M; yellow arrow), in the optic nerves and pathway $(\mathrm{N}, 0)$, in the acoustic pores $(\mathrm{P})$ and geniculate ganglia, as well as global $(Q, R)$ and pre-pontine crura pachymeningeal enhancement.

Case 6. (S to V) A 45-year-old male which developed atypical pneumonia by SARS-CoV-2 and showed severe respiratory distress. After one month of recovery, he developed headaches, blurred vision, dizziness, and vertigo. High IgM and low IgG anti-SARS-CoV-2 antibody titers were detected. At MRI, after gadolinium administration it was observed a slight enhancement of the cochlear nerves and vestibular complexes ( $\mathrm{S}$ and $\mathrm{T}$, yellow circles), as well as the right geniculate ganglia. There was a marked enhancement in the cavernous sinuses and rostral to the cortices of the temporal lobes (U and V). 
[49]. Another neuroimaging study by Katal et al. included 116 COVID-19 patients; from them, only 90 had neuro-COVID-19 symptoms. In this group, 37 patients had normal brain CT o MRI imaging results, while several abnormalities were found in the rest, including vascular thrombosis, cortical signal abnormalities, hemorrhage, hemorrhagic PRES, acute necrotizing encephalopathy (ANE), meningitis/encephalitis, and acute disseminated encephalomyelitis (ADEM) [55]. In a French study, Chougar et al. described 1176 patients suspected of COVID-19 infection. From these, 308 patients developed neurological symptoms and 73 met the inclusion criteria. The male population predominated, $58.9 \%$ of patients showed abnormal MRI findings 2-4 weeks after symptom onset: $23.3 \%$ suffered from acute infarct, $1.4 \%$ from deep venous thrombosis, $11.3 \%$ from microhemorrhages, $47.7 \%$ from perfusion abnormalities, $4.1 \%$ from cytotoxic lesions of the corpus callosum, $5 \%$ from multifocal white matter-enhancing lesions, and 5\% from basal ganglia abnormalities [56]. In another study by Kremer et al. including 37 French patients, white matter abnormalities in the medial temporal region (like viral or autoimmune encephalitis) were the most frequent findings $(43 \%)$, followed by diffuse, non-confluent multifocal white matter hyperintense lesions associated to hemorrhages $(30 \%)$, and white matter microhemorrhages (24\%) but also gyral, meninge$\mathrm{al}$, and perineural enhancements been described in children and adult COVID-19 patients [50-58]. The pandemic exacerbated pre-existing deficiencies in the health systems of LMIC. Sanitary measures (mask and hand washing), as well as vaccination programs, are essential to control the pandemic. As of November 1, 2021, 7,116,206.54 vaccine doses had been administered worldwide [47].

\section{CONCLUSIONS}

Neglected and (re-)emerging infections of the CNS constitute important public health concerns, particularly in LMIC, where poverty, inequality, and other socioeconomic problems contribute to their increased incidence. The great information gap regarding surveillance reporting of several neglected infections in LMIC indicate a high risk for adequate early identification of outbreaks. A more comprehensive approach requires improving housing conditions, sanitary practices, and ac- cess to clean drinking water, as well as reducing the number of arthropod vectors for these infections and ensuring the access to vaccines in the open population to control them in an effective manner.

\section{Acknowledgements}

The authors thank to Dr. Carrillo-Alvarez Santiago, fellow from neuroimaging department, for his help in editing of images, and Juan Francisco Rodriguez for copy-editing this manuscript.

\section{Author contributions}

Study concept and design: GC, PS, EP. Data acquisition: GC, PS, EP, ELF, IPR, RR, RDDH. Manuscript drafting: GC, PS, EP, ELF, IPR, RR, RDDH. Critical revision of the manuscript for important intellectual content: GC, PS, EP, RR. Study supervision. GC, PS, EP, ELF, IPR, RDDH.

\section{Funding statement}

This research received no specific grant from any funding agency in the public, commercial, nor not-for-profit sectors.

\section{Competing interests statement}

The authors declare that no conflict of interest exists.

\section{REFERENCES}

[1] Furuse Y. Analysis of research intensity on infectious disease by disease burden reveals which infectious diseases are neglected by researchers. Proc Natl Acad Sci USA. 2019; 116 (2), 478-83.

[2] GBD 2016 DALY and HALE collaborators global, regional, and national disability-adjusted life-years (DALYs) for 333 diseases and injuries and healthy life expectancy (HALE) for 195 countries and territories, 1990-2012: A systematic analysis for the Global Burden of Disease Study 2016. Lancet. 2017; 390 (10100), 1260344.

[3] World Population Ageing 2020 Highlights. https:/ / www.un.org/development/desa/pd/sites/www. un.org/development.desa.pd/files/undesa_pd-2020_ world_population_ageing_highlights.pdf. [accessed November 1, 2021].

[4] World Population 2019. At https://population. un.org/wpp/Publications / Files/WPP2019-wallchart. pdf. [accessed November 1, 2021].

[5] Kimura-Hayama ET, Higuera JA, Corona-Cedillo R, et al. Neurocysticercosis:radiologic-pathologic correlation. Radiographics 2010; 30 (6), 1705-19 
[6] World Health Organization epidemiology 2015. https://www.who.int/taeniasis/epidemiology/en/. [accessed on November 1, 2021].

[7] Gripper LB, Welburn SC. Neurocysticercosis infection and disease. A review. Acta trop. 2017; 166, 218-24. [8] Raibagkar P, Berkowitz AL. The many faces of neurocysticercosis. J Neurol Sci. 2018; 390, 75-6.

[9] Garcia HH, O’Neal SE, Noh J, Handali S. Cysticercosis Working Group in Peru. Laboratory diagnosis of neurocysticercosis (Taenia solium). J Clin Microbiol. 2018; 56 (9), e00424-28.

[10] Bobes RJ, Hernández M, Marquez C, et al. Subarachnoidal and intraventricular human neurocysticercosis: application of an antigen detection assay for the diagnosis and follow-up. Trop Med Int Health. 2006; 11 (6), 943-50.

[11] Sahu PK, Satpathi S, Behera PK, Mishra SK, Mohanty S, Wassmer SC. Pathogenesis of cerebral malaria: new diagnostic tools, biomarkers, and therapeutic approaches. Front Cell Infect Microbiol. 2015; 5, 75.

[12] Moxon CA, Gibbins MP, McGuiness D, Milner DA Jr, Marti M. New insights into malaria pathogenesis. Annu Rev Pathol. 2020; 15, 315-43.

[13] World Malaria Report 2020. Available at https:// www.mmv.org/sites/default/files/uploads / docs / publications/World_Malaria_Report_2020.pdf. [accessed November 1, 2021].

[14] Pan American Health Organization/ World Health Organization. Topics. https://www.paho.org/en/malaria. [accessed November 1, 2021].

[15] Luzolo AL, Ngoyi DM. Cerebral malaria. Brain Res Bull. 2019; 145, 53-8.

[16] Mohanty S, Benjamin LA, Maihi M, et al. Magnetic resonance imaging of cerebral malaria patients reveals distinct pathogenic processes in different part of the brain. mSphere. 2017; 2 (3), 300193-17.

[17] Farahtaj F, Alizadeh L, Gholami A, et al. Natural infection with rabies virus: A Histopathologica and Immunohistochemical study of human brains. Osong Public Health Res Perspect. 2019; 10 (1), 6-11.

[18] Hampson K, Coudeville L, Lembo T, et al. Global Alliance for Rabies Control Partners for Rabies Prevention. Estimating the global burden of endemic canine rabies. PLoS Negl Trop Dis. 2015; 9 (4), e0003709.

[19] WHO Expert Consultations on rabies TRS n¹012,2017. https: / / www.who.int/activities/improving-data-on-rabies / rabies-epidemiology-and-burden. [accessed on November 1, 2021].

[20] Hemachudha T, Ugolini G, Wacharapluesadee S, Sungkarat W, Shuangshoti S, Laothamatas J. Human rabies: neuropathogenesis, diagnosis and management. Lancet Neurol. 2013; 12 (5), 498-513.

[21] Jackson AC. Human rabies: a 2016 update. Curr Infect Dis Rep. 2016; 18 (11), 38.

[22] Co SJ, Mackenzie IR, Shewchuk JR. Rabies encephalitis. Radiographics. 2015; 35 (1), 235-8.
[23] World Malaria Report 2020. https://who.int/ teams / global-malaria-programme/reports / worldmalaria-report-2020/. [accessed on November 1, 2021]. [24] CDC. West Nile Virus Final cumulative maps and Data. Available at https://www.cdc.gov/westnile/ statsmaps/finalmalpsdata/index.html. [accessed November 1, 2021].

[25] Popescu CP, Florescu SA, Hasbun R, et al. Prediction of unfavorable outcomes in West Nile virus neuroinvasive infection- Result of a multinational ID-IRI study. J Clin Virol. 2020; 122, 104213.

[26] Castro-Jorge LA. Siconelli MJL, Ribeiro BDS, et al. West Nile virus infection are here! Are we prepared to face another flavivirus epidemic? Rev Soc. Bras Med Trop. 2019; 52, e20190089.

[27] Cadena AM, Fortune SM, Flynn JL. Heterogeneity in tuberculosis. Nat Rev Immunol. 2017; 17 (11), 691-702. [28] WHO. Global tuberculosis report 2020. Available at https:/ / www.who.int/teams/global-tuberculosis-programme/tb-reports/global-tuberculosis-report-2020. [accessed November 1, 2021].

[29] Schaller MA, Wicke F, Foerch C, Weidauer S. Central nervous system tuberculosis: etiology, clinical manifestation and neuroradiologic features. Clin Neuroradiol. 2019; 29 (1), 3-18.

[30] Marais S, Thwaites G, Schoeman JF, et al. Tuberculous meningitis: a uniform case definition for use in clinical research. Lancet Infect Dis. 2010; 10 (11), 803-12. [31] Pormohammad A, Riahi SM, Nasiri MJ, et al. Diagnostic test accuracy of adenosine deaminase for tuberculous meningitis: A systematic review and metaanalysis. J Infect. 2017; 74 (6), 545-54.

[32] Garg RK, Malhotra HS, Jain A. Neuroimaging in tuberculous meningitis. Neurol India 2016; 64 (2), 219-27. [33] Azeemudin M, Alvi A, Sayani R, et al. Neuroimaging findings in tuberculosis: A single center experience in 559 cases. J Neuroimaging. 2019; 29 (5), 657-68.

[34] Remesar S, Diaz P, Venzal JM, et al. Longitudinal Study of Infection with Borrelia spp. in Questing Ticks from North-Western Spain. Vector-Borne Zoonotic Dis. 2019,19 (11),785-92.

[35] WHO, Europe 2006. Lyme borreliosis in Europe: influences of climate and climate change, epidemiology, ecology, and adaptation measures. https: / /www.euro. who.int/_data/assets/pdf/0006/96819/E89522.pdf. [accessed on November 1, 2021].

[36] Schwartz AM, Hinckley AF, Mead PS, Hook SA, Kugeler KJ. Surveillance for Lyme Disease -United States, 2008-2015. MMWR Surveill Summ. 2017; 66 (22), $1-12$.

[37] Gordillo-Perez G, Solorzano F, Cervantes-Castillo A, et al. Lyme neuroborreliosis is a severe and frequent neurological disease in Mexico. Arch Med Res. 2018; 49 (6), 399-404.

[38] Brodzínski S, Nasierowski T. Psychosis in Borrelia burgdorferi infection - part 1: epidemiology, pathogen- 
esis, diagnosis, and treatment of neuroborreliosis. Psychiatry Pol. 2019; 53 (3), 629-40.

[39] Halperin JJ. Neuroborreliosis. Neurol Clin. 2018; 36 (4), 821830.

[40] Carmo RLD, Alves-Simão AK, Amaral LLFD, et al. Neuroimaging of emergent and re-emergent infections. Radiographics. 2019; 39 (6), 1649-71.

[41] Cui J, Li F, Shi ZL. Origin and evolution of pathogenic coronaviruses. Nat Rev Microbiol. 2019; 17 (3), 181-92.

[42] Ding S, Liang TJ. Is SARS-CoV-2 also an enteric pathogen with potential fecal-oral transmission? A COVID-19 virological and clinical review. Gastroenterology. 2020; 159 (1), 53-61.

[43] Jin T, Yang H, Ji W, et al. Virology, epidemiology, pathogenesis, and control of COVID-19. Viruses. 2020; $12(4), 372$.

[44] de Wit E, van Doremalen N, Falzarano D, Munster VJ. SARS and MERS: recent insights into emerging coronaviruses. Nat Rev Microbiol. 2016; 14 (8), 523-34.

[45] Hoffmann M, Kleine-Weber H, Schroeder S, et al. SARS-CoV-2 cell entry depends on ACE2 and TMPRSS2 and is blocked by a clinically proven protease inhibitor. Cell 2020; 181 (2), 271-80.

[46] Song E, Zhang C, Israelow B, et al. Neuroinvasion of SARS-CoV-2 in human and mouse brain. J Exp Med. 2021; 218 (3), e20202135.

[47] Coronavirus Resource Center. John Hopkins University of Medicine. https://coronavirus.jhu.edu/ map.html. [accessed November 1, 2021].

[48] WHOCoronavirus (COVID-19) Dashboard.https:/ / covid19.who.int. [accessed on November 1, 2021].

[49] Lin E, Lantos JE, Strauss SB, et al. Brain imaging of patients with COVID-19: Findings at an Academic Institution during the height of the outbreak in New
York City. AJNR Am J Neuroradiol. 2020; 41 (11), 2001-8. [50] Revzin MV, Raza S, Warshawsky R, et al. Multisystem imaging manifestations of COVID-19, Part 1: Viral pathogenesis and pulmonary and vascular system complications. Radiographics. 2020; 40 (6), 1574-99.

[51] Revzin MV, Raza S, Warshawsky R, et al. Multisystem imaging manifestations of COVI-19, Part 2: From cardiac complications to pediatric manifestations. Radiographics. 2020; 40 (7), 1866-92.

[52] Mao L, Jin H, Wang M, et al. Neurologic manifestations of hospitalized patients with coronaviruses disease 2019 in Wuhan, China. JAMA Neurol. 2020; 77 (6), 683-90.

[53] Sheraton M, Deo N, Kashyap R, Surani S. A review of neurological complications of COVID-19. Cureus. 2020; 12 (5), e8192.

[54] Brouwer MC, Ascione T, Pagliano P. Neurologic aspects of COVID-19: a concise review. Infez Med. 2020; (Suppl. 1), 42-5.

[55] Katal S, Balakrishnan S, Gholamrezanezhad A. Neuroimaging and neurologic findings in COVID-19 and other coronavirus infection: a systematic review in 116 patients. J Neuroradiol. 2021; 48 (1), 43-50.

[56] Chougar L, Shor N, Weiss N, et al. Retrospective observational study of brain MRI findings in patients with acute SARS-CoV-2 infection and neurologic manifestations. Radiology. 2020; 297 (3), E313-23.

[57] Kremer S, Lersy F, de Sèze J, et al. Brain MRI findings in severe COVID-19: a retrospective observational study. Radiology. 2020; 297 (2), E242-51.

[58] Lindan CE, Mankad K, Ram D, et al. Neuroimaging manifestations in children with SARS-CoV-2 infection: a multinational, multicentre collaborative study. Lancet Child Adolesc Health. 2021; 5 (3), 167-77. 